\title{
Study and realization of control system for venous transfusion
}

\section{automatic dispensing apparatus}

\author{
Lijian Zhang ${ }^{1, a}$, Haina Cui ${ }^{2, b}$, Tingting Wang ${ }^{3, c}$ \\ ${ }^{1}$ College of Electrical Engineering, Binzhou Polytechnic,Shandong,256600,China \\ ${ }^{2}$ College of Nursing, Binzhou Polytechnic,Shandong,256600,China \\ ${ }^{3}$ Bohai Piston Co.,Ltd, Binzhou,Shandong,256600,China \\ axianruibrother@163.com, b41350992@qq.com, c1653226361@qq.com
}

Keywords: Pollution, Infusion, Automatic, Control

\begin{abstract}
Many disadvantages exist in human intravenous infusion preparation process, with cross contamination. Effectively prevent intravenous fluids should be dispensed pollution, this paper introduces the automatic dispensing device structure and working principle of the control system, the experimental results show that this system operation is simple, dissolution and improve the quality of the infusion preparation with high efficiency.
\end{abstract}

\section{Introduction}

Intravenous transfusion is one of the common treatment measures for clinical treatment and rescue of patients. The drug directly enters human blood by intravenous drip, and has a high absorption rate and quick effect.. Therefore, the intravenous drip of drug indicators should be strict requirements, once the quality of the problem will directly affect human health, and even life-threatening.

At present, each big hospital intravenous infusion preparation center, occupy large area and additional facilities investment, drug preparation have medical personnel manual preparation, medical staff labor intensity big, in the process of operation exists many disadvantages, for example, visible particles,

The phenomenon of the dissolved particles and the microorganism and other factors pollution liquid is common.

Dispensing robot Canada intelligent hospital systems, research on trial in hospital, preparation of high precision, but covers an area of area is larger, the weight of a large, some execution mechanism design is too complicated. Although there are other companies, such as MDS and Panasonic also have involved in the field, but there is no product available, China is still a blank in the field.

\section{Dispensing tester}

In this paper, the design of the venous transfusion automatic preparation instrument with PLC as the controller and the touch screen to do as the host computer, take a step motor control technology, under the full closed sterile environment, with liquid, fully automated operation.

The instrument can realize the liquid in the closed condition, by scanning gun hospital with liquid electronic prescription, medical personnel will be bottles of liquid medicine is put in the specified location, drug under the function of automatic push / ceiling system, infusion bottle to drug injection in the medicine bottle, after a specified number of repeated preparation, until the 
photometric meter of liquor turbidity value detection qualified, configured liquid transported back to the original liquid dropping bottle, in order to achieve venous transfusion automatic configuration and monitoring.

\section{Dispensing apparatus}

Mainly by the electrical control system, piping system, networking system and accessories.

\section{Electrical control system}

Dispensing apparatus control system is mainly composed of Beijing KunLun Winton, PLC, touch screen scanning gun, spectrophotometer, sensor, stepper motor and drive equipment.

\section{PLC control system}

PLC accepts the signal of the photoelectric sensor, control two stepper motor coordinated motion, completed the dispensing system automatically push liquid and water absorption and drug dissolution is the power source of the dispensing system for Chinese medicine liquid.

PLC input point is mainly of speed-up in the origin of the motor and limit position sensor signal, single runway into the motor start and stop signal and emergency stop signal. The output signal of PLC contains the running state and output indicator system PTO two stepper motor etc..

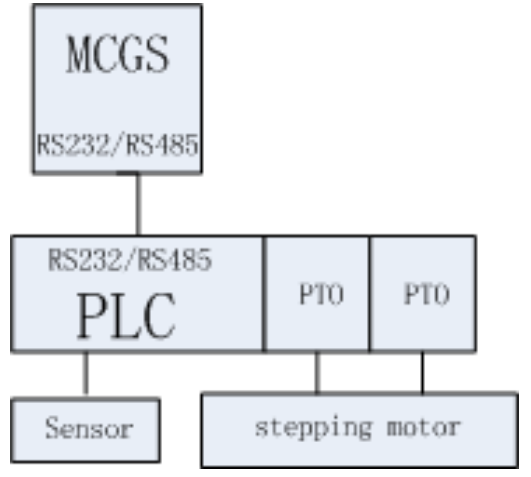

Fig.1 control system

\section{Man-machine interface}

Using the man-machine interface as the monitoring system, the real-time data of PLC is displayed, recorded, stored, processed, and thus meets all kinds of monitoring requirements.. Considering the system compatibility and expansibility, the Beijing Tongtai KunLun touch screen.

The interface includes user right interface, parameter setting interface, automatic monitor screen, database interface, alarm picture and so on..

In the automatic monitoring picture, the system starts the stop button, the change disposition strategy, displays the pharmacy stock, the display each part of the movement condition and so on.

User rights interface, with access to the medical staff before entering the appropriate interface, password error input three times, will lock screen.

The parameters of the interface, the communication parameters of PLC and spectrophotometer, the running speed of the stepping motor and the washing of the pipeline, etc..

The database interface, the compatibility between the drug compatibility taboo table, after the pharmacist audit through the query compatibility tabu list database to determine the incompatibility taboo. Add the basic information and medication records to the system database, and can be readily consulted in the future treatment, and with reference to the previous medication to determine the 
reasonable treatment plan.

\section{Networking system}

The system should have the ability of network interconnection. Through the network, doctors directly to the prescription online entry, pharmacists can through the electronic prescription online audit, and through the examination of the prescription hair to put medicine personnel, prepare corresponding drugs and syringes, and other equipment, dispensing staff through the human-computer interaction will send the items put into the system. All the information is transmitted on the network, which improves the efficiency of the transmission, and the network is also

The Internet can achieve remote monitoring and maintenance system for dispensing apparatus.

\section{Liquid circulation system}

Drip bottle, disposable syringes, solenoid valve, and designed the line of enclosed with liquid circulation system, etc. Circulation system of electromagnetic valve, sensor arrangement is shown in figure 2. After fully dissolved in drip bottle of drugs, to get the rooms for patients to use. Solenoid valve in accordance with the drug dissolution process, on and off with liquid circulation system in real time.

Closed loop with liquid pipeline system, including cross needles (102), injection pump connecting line (103), six needle (104), side road connecting head (105), end seal (106) five parts; Four-way needle (102) through a hose and injection pump connecting line (103), side road connecting head (105), six needle (104) connection, terminal idle end by the end seal (106) block form solvent and liquid circulation channels. Four-way needle (102) for one or two, solvent side $\operatorname{road}(101 \mathrm{~A})$ and solution $(101 \mathrm{~B})$ side road in six general needle $(104)$ on the same side or different sides with one or two cross needles (102) connected to constitute A closed two-way cycle with liquid line (A) or closed unidirectional cycle with liquid pipeline (B), (C), on both sides of the road between six passed needle (104) in A loop inside the bottle, and formed in the two way under the action of the injection pump or uniflow two-way flow. The infusion bottle/bag solvent liquid by solvent side of the road (101 a) infusion into my bottle of powder injection drug dissolved, then through liquid side road (101 b) will dissolve the solution back to the original infusion bottle/bag or sent to another infusion bottle/bag, can through the side of the road linked end to the water bottle (105), the liquid directly sent to the infusion bottle/bag, realize the automation of venous transfusion in the closed state preparation.
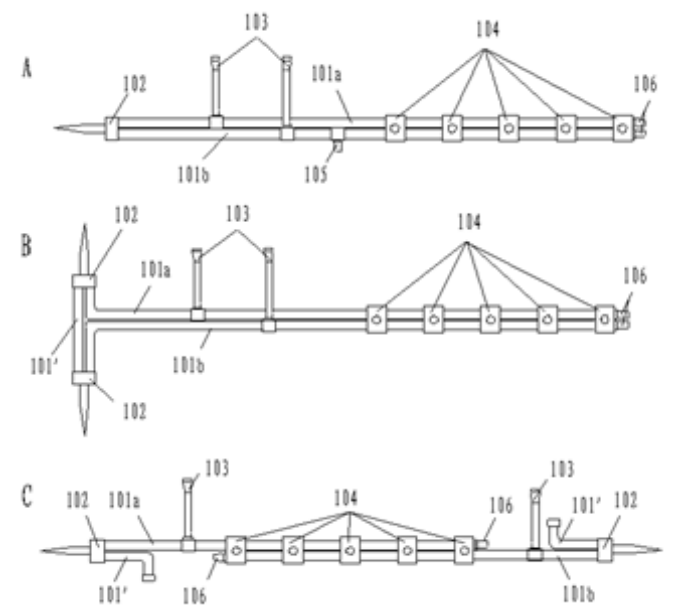

Fig.2: The Piping 


\section{Spectrophotometer and scanner}

By calculating the absorbance of the liquid, the spectrophotometer determines whether the solid particles are fully dissolved.. And PLC by fixed format serial communication, mutual access to effective data information.

Laser scanner is mainly used for scanning drug contraceptives and other one-dimensional bar code information and transmits it to the computer, so that the operating personnel to identify drug review etc..

\section{Attached parts}

In addition to the above main system, the automatic dispensing equipment is composed of base, supporting frame, waste containers, and electrical control box and other accessory parts such as the whole equipment.

\section{PLC control program}

According to the design of the pipeline and the action, the logic control of the solenoid valve is written by PLC to realize the sequence of the solenoid valve, and realize the on-off control of the pipeline.. Figure 3 is a part of the program with EasyLad software.

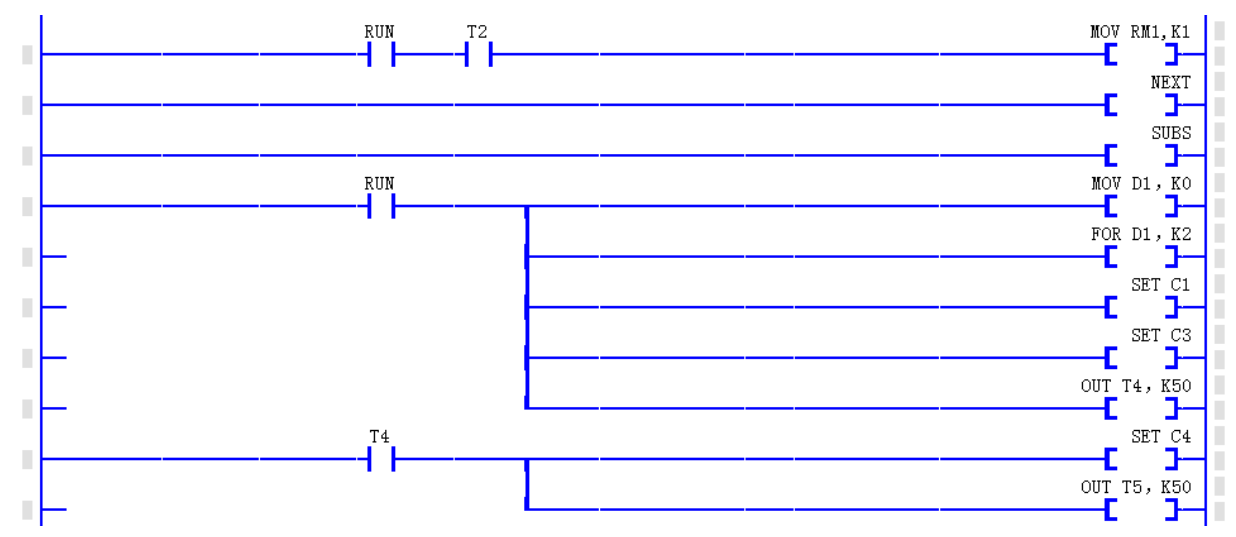

Fig.3: The Program

\section{Experiment}

\section{Preparation}

At a certain mixing center, the nurse was divided into two groups, the number was three, the average age was 35 years old..

\section{Method}

The drug was divided into 10 batches, and the mixing speed of the two groups and the turbidity of the drug particles and the fatigue of the personnel were observed..

\section{Effect evaluation criteria}

Complete dispensing time as short as possible, aseptic operation, high solubility, no obvious change of interpersonal relationship.

\section{Statistical methods and results}

The data were represented by the mean of the standard deviation, and the t test was used to compare the difference between the groups. 
Table 1 Comparison of experimental results

\begin{tabular}{|l|l|l|l|l|}
\hline Group & time & transmittance & $\mathrm{P}$ & $\mathrm{T}$ \\
\hline Manual & 13.5 & $<34.3$ & $>0.05$ & 0.25 \\
\hline $\begin{array}{l}\text { Dispensing } \\
\text { apparatus }\end{array}$ & 9.4 & $>45.7$ & $<0.05$ & 0.17 \\
\hline
\end{tabular}

\section{Conclusion}

Average dose, work number between group differences were not statistically significant $(\mathrm{P}>$ 0.05); dispensers to complete the difference between the groups of time have statistical significance, $\mathrm{P}<0.05$, dispensing instrument for completion time compared with manual group decreased significantly, ahead of time about the $4.1 \mathrm{~min}$ complete dispensing and sent to the ward. The dispensing instrument for the completion time of calculation, it can reduce 2 of people go to work every day. The dispenser to adhere to the principles of aseptic dispensing, accurate dosage, no special change of interpersonal relationship.

\section{Acknowledgement}

This research was financially supported by Colleges and universities in shandong province department of education scientific research and development projects.

Project name: intravenous infusion of the control system of automatic dispensing apparatus development with item number: J14LB57.

\section{References}

[1]quiet early,Liu Yuxin, application of [J]. technology.2012 Chen Wei.BAWJ pageautomatic dispensing system in coal preparation plant in Xiaoming mine (29)

[2]Huang Caixia,Qi Jingjing,Huang Libin.Automatic measurement and control systemis designed with.2013[J]. computer Petrochemical agents based on PLC (21)

[3]Wang Chao. Research on [3] [J].2013 process automation instrumentation is a kind of new intelligent dispensing equipment (7)

[4] Guo Liping [J].2013. To improve the practice of clinical medicine in pediatric infusionliquid dispensing process technology (2)

[5]Liu Qiuyuan, Li Hongcui, Li Jianhua.Discussion on reducing dispensing needlecutting method of rubber stopper crumbs [J]. Jilin.2013 (32) 